\title{
Características anatômicas do plexo braquial de tamanduá-mirim (Tamandua tetradactyla Linnaeus, 1758)
}

\author{
Anatomical characteristics of the brachial plexus of lesser anteater ("Tamandua \\ tetradactyla” Linnaeus, 1758)
}

CRUZ, Géssica Ariane de Melo; ADAMI, Marta ${ }^{2 *}$; ALMEIDA, Ana Elisa Fernandes de Souza $^{2}$; SILVA, Érica Augusta dos Anjos Cerqueira da ${ }^{1}$; FARIA, Márcia Maria Magalhães Dantas de ${ }^{2}$; PINTO, Maria das Graças Farias ${ }^{2}$; SILVA, Ricardo Diniz Guerra e

\footnotetext{
${ }^{1}$ Universidade Federal da Bahia, Escola de Medicina Veterinária e Zootecnia, Salvador, Bahia, Brasil.

${ }^{2}$ Universidade Federal da Bahia, Escola de Medicina Veterinária e Zootecnia, Departamento de Anatomia Veterinária, Salvador, Bahia, Brasil.

*Endereço para correspondência: madami@ufba.br
}

\section{RESUMO}

Objetivou-se com este trabalho identificar a origem, composição e os nervos do plexo braquial de tamanduá-mirim. Dois cadáveres foram cedidos pelo Centro de Triagem de Animais Silvestres (CETAS) Chico Mendes, Salvador, Bahia e a execução do projeto foi autorizada pelo Sistema de Autorização e Informação em Biodiversidade (SISBIO - IBAMA) n²0268-1 (29/05/2009). Os resultados foram descritos, esquematizados e fotografados, e os termos anatômicos adotados foram os preconizados pelo International Committee on Veterinary Gross Anatomical Nomenclature. $\mathrm{O}$ plexo braquial recebeu contribuição dos segmentos medulares C5; C6; C7; C8 e T1, com a formação de três troncos: cranial (C5 e C6), médio (C7) e caudal (C8 e T1) que originaram os nervos derivados do plexo, com exceção do nervo cutâneo medial do antebraço que teve origem unissegmentar (T1). O plexo braquial de tamanduá-mirim apresentou origem, composição e formação dos nervos periféricos semelhantes ao observado em grande parte de outros mamíferos domésticos e silvestres.

Palavras-chave: anatomia, animal silvestre, plexo braquial, sistema nervoso.

\section{SUMMARY}

The aim of this study was to identify the origin, composition and the nerves from the brachial plexus of the lesser anteater. Two cadavers were given by the Wild Animals Screening Center (CETAS Chico Mendes), Salvador city, Brazil and the execution of the project was authorized by the System of Authorization and Information in Biodiversity (SISBIO - IBAMA) n²0268-1 (29/05/2009). The results were described, schematized and photographed, and the anatomic terms adopted were the ones recommended by the International Committee on Veterinary Gross Anatomical Nomenclature. The brachial plexus received contribution from the spinal cord segments C5; C6; C7; C8 and T1 with the formation of three trunks: cranial (C5 and C6), median (C7) and caudal (C8 and T1) which originated the nerves derived from the plexus, except the antebrachial medial cutaneous nerve that was of a monosegmental origin. The brachial plexus of the lesser anteater presented origin, composition and formation of peripheral nerves similar to the observed in several other domestic and wild mammals.

Keywords: anatomy, brachial plexus, nervous system, wild animal. 
Rev. Bras. Saúde Prod. Anim., Salvador, v.13, n.3, p.712-719 jul./set., 2012 http://www.rbspa.ufba.br ISSN 15199940

\section{INTRODUÇÃO}

Tamandua tetradactyla, mamífero da Ordem Pilosa, popularmente conhecido como tamanduá-mirim ou tamanduá-de-colete, faz parte da Família Myrmecophagidade que inclui o Gênero Tamandua e quatro espécies de tamanduás (MEDRI et al., 2006). Ocorre que em vários países das Américas e no Brasil aparece em todos os biomas. Espécie pertencente à Ordem Pilosa e Família Myrmicophagidae, apresenta índole pacífica, contudo, se ameaçado adota comportamento de defesa que é comum aos animais dessa Ordem. Nesse sentido, posiciona-se de forma ereta sob um tripé apoiado com as duas pernas e a cauda e deixa as garras torácicas livres (MEDRI et al., 2006; MIRANDA \& COSTA, 2006).

A Ordem Xenarthra possuía uma diversidade de espécies, porém hoje a maioria encontra-se extinta e algumas ameaçadas de extinção ou vulneráveis, como determinadas espécies de preguiças e tamanduás. Felizmente, o tamanduámirim está classificado pela International Union for Conservation of Nature IUCN- como pouco preocupante (IUCN, 2011).

O plexo braquial já foi descrito em diversas espécies domésticas: caprino, ovino, bovino, suíno e equino (MAGILTON et al., 1968); silvestres: capivara (FIORETTO et al., 2003), mocó (SANTANA et al., 2003), macaco-prego (RIBEIRO et al., 2005), veado (MELO et al., 2007), cateto (MOURA et al., 2007), paca (SCAVONE et al., 2008) e exóticas: porcoespinho (AYDIN, 2003) e chinchila (GAMBA et al., 2007). Em xenartros são raras as referências sobre investigações do sistema nervoso. No que diz respeito às pesquisas nesse contexto, podem ser encontrados dados sobre a constituição do plexo braquial em preguiças (BIELIK, 1937; AMORIM et al., 2003). Entretanto, especificamente, sobre tamanduás não existem informações sobre a formação do plexo.

Objetivou-se descrever o plexo braquial de tamanduá-mirim em sua origem, composição e nervos derivados. As informações contribuirão para o estabelecimento de padrões morfofuncionais que poderão ser utilizados para análises comparativas com as demais espécies e com a adoção de medidas que visem proporcionar $\mathrm{o}$ bem-estar animal e a preservação da espécie.

\section{MATERIAL E MÉTODOS}

Duas fêmeas (adulto e filhote), mortas por causas naturais, foram cedidas pelo Centro de Triagem de Animais Silvestres Chico Mendes (CETAS), Salvador, Bahia, para o Departamento de Anatomia Veterinária da Escola de Medicina Veterinária e Zootecnia, Universidade Federal da Bahia (UFBA). A pesquisa foi autorizada pelo Sistema de Autorização e Informação em Biodiversidade (SISBIO) n'20268-1 (29/05/2009) do Instituto Brasileiro do Meio Ambiente e dos Recursos Naturais Renováveis (IBAMA). Os animais foram fixados com solução de formaldeído a $10 \%$ injetada via artéria carótida comum e, posteriormente, procedeu-se o rebatimento da pele e a dissecação dos músculos da região dorsal da coluna cervical para a exposição das vértebras, cujos corpos foram retirados e a medula espinhal exposta. Em seguida, os segmentos medulares com os respectivos nervos espinhais que originam o plexo braquial e os nervos periféricos nos dois antímeros foram dissecados e identificados com auxílio de lupa estereoscópica (PZO Labimex). A terminologia adotada baseouse no preconizado pelo International 
Rev. Bras. Saúde Prod. Anim., Salvador, v.13, n.3, p.712-719 jul./set., 2012 http://www.rbspa.ufba.br ISSN 15199940

Commitee on Veterinary Gross Anatomical Nomenclature (2005).

\section{RESULTADOS E DISCUSSÃO}

O tamanduá-mirim possui sete vértebras cervicais, com a emergência do primeiro par de nervo espinhal cervical entre o occipital e o atlas. O plexo braquial (PB) derivou-se a partir do quinto, sexto, sétimo e oitavo segmentos medulares cervicais e primeiro torácico, que corresponderam aos respectivos nervos espinhais relacionados: $\mathrm{C} 5$ a C8 e T1. Os nervos espinhais originaram três troncos: cranial (C5 e C6), médio (C7) e caudal (C8 e T1), conforme as Figuras 1 e 2.

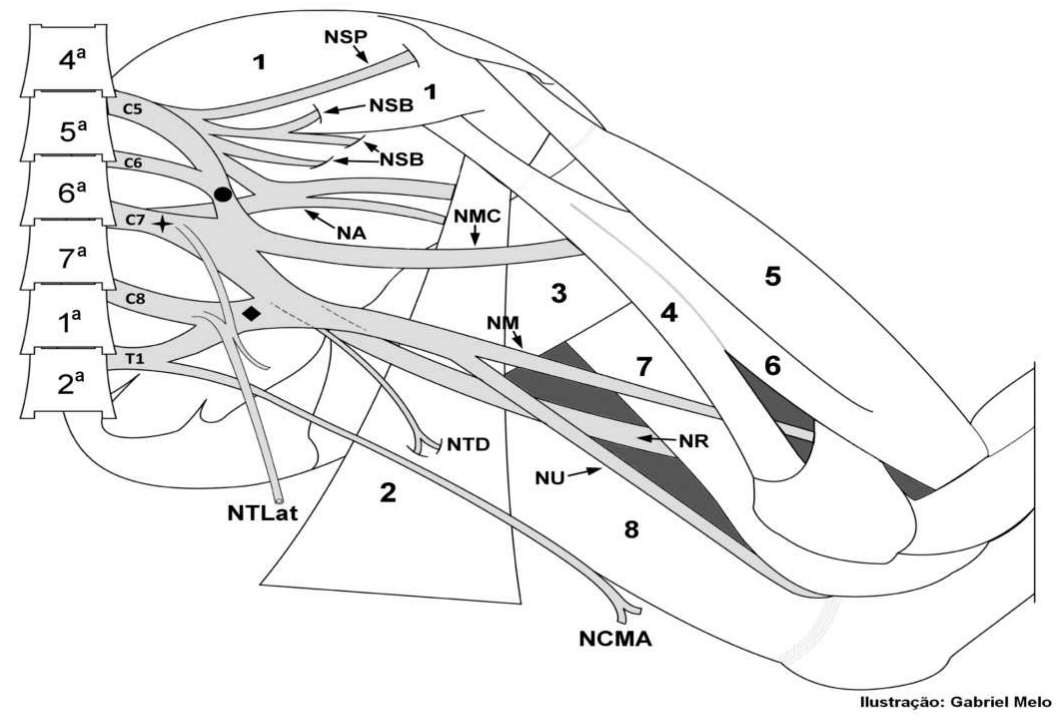

Figura 1. Representação esquemática da vista medial do plexo braquial de tamanduá-mirim (Tamandua tetradactyla): Origem, composição dos troncos e nervos resultantes. Vértebras: $4^{\mathrm{a}}$ quarta vértebra cervical, $5^{\mathrm{a}}$ quinta vértebra cervical, $6^{\mathrm{a}}$ sexta vértebra cervical, $7^{\mathrm{a}}$ sétima vértebra cervical, $1^{\mathrm{a}}$ primeira vértebra torácica, $2^{\mathrm{a}}$ segunda vértebra torácica. Nervos espinhais: $\mathrm{C} 5-5^{\circ}$ nervo cervical, C6- $6^{\circ}$ nervo cervical, C7$7^{\circ}$ nervo cervical, $\mathrm{C} 8-8^{\circ}$ nervo cervical, T1- $1^{\circ}$ nervo torácico Troncos: cranial $(\bullet)$, médio $(\boldsymbol{\downarrow})$, caudal $(\bullet)$. Músculos: 1- subescapular; 2- grande dorsal; 3- redondo maior; 4- coracobraquial; 5- bíceps braquial (porção curta) 6bíceps braquial (porção longa); 7- tríceps braquial cabeça medial; 8- tríceps braquial cabeça longa. Nervos: supraescapular (NSP), subescapulares (NSB), axilar (NA), mediano (NM), musculocutâneo (NMC), radial (NR), cutâneo medial do antebraço (NCMA), ulnar (NU), toracodorsal (NTD), torácico lateral (NTLat) 


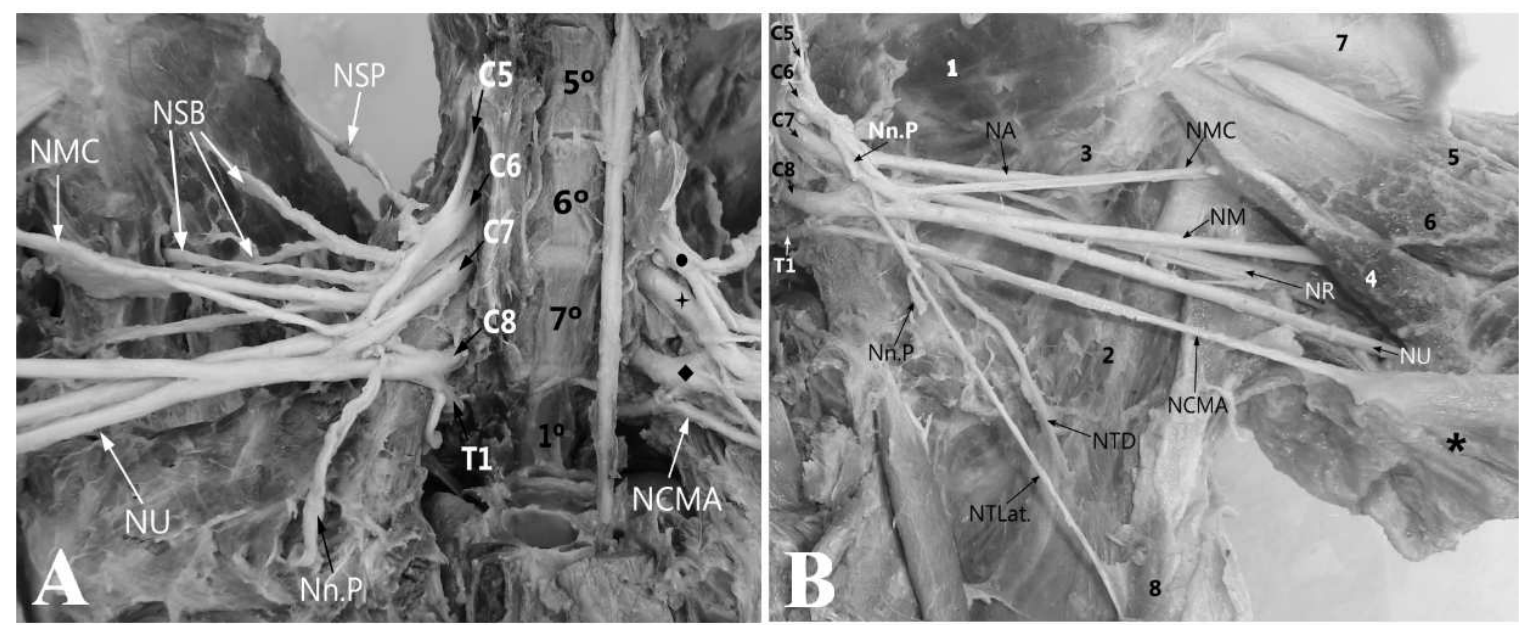

Figura 2 (A-B). Vista medial do plexo braquial de tamanduá-mirim (Tamandua tetradactyla): Origem, composição dos troncos e nervos resultantes. Vértebras: $5^{\mathrm{a}}$ quinta vértebra cervical, $6^{\mathrm{a}}$ sexta vértebra cervical, $7^{\mathrm{a}}$ sétima vértebra cervical, $1^{\text {a }}$ primeira vértebra torácica. Nervos espinhais: $\mathrm{C} 5-5^{\circ}$ nervo cervical, $\mathrm{C} 6-6^{\circ}$ nervo cervical, $\mathrm{C} 7-7^{\circ}$ nervo cervical, C8- $8^{\circ}$ nervo cervical, T1- $1^{\circ}$ nervo torácico. Troncos: cranial $(\bullet)$, médio $(\boldsymbol{+})$, caudal $(\diamond)$. Nervos: supraescapular (NSP), subescapulares (NSB), axilar (NA), mediano (NM), musculocutâneo (NMC), radial (NR), cutâneo medial do antebraço (NCMA), ulnar (NU), toracodorsal (NTD), torácico lateral (NTLat), peitorais (Nn.P). Músculos: 1- subescapular; 2- grande dorsal; 3- redondo maior; 4coracobraquial; 5- bíceps braquial (porção curta) 6- bíceps braquial (porção longa); 7- tríceps braquial cabeça medial; 8- tríceps braquial cabeça longa

A origem do plexo assemelhou-se ao encontrado em suínos, koala (Phascolarctos cinereus), rato, cobaia, coelho (PATERSON, 1887), cateto (MOURA et al., 2007) e alguns primatas (BOOTH et al., 1997; CRUZ \& ADAMI, 2010) e diferiu do observado em mocó, no qual, na maioria dos animais estudados, o PB recebeu contribuição dos segmentos C6 a T2 (SANTANA et al., 2003), na capivara de C4 a T1 (FIORETTO et al., 2003) e no lobo-marinho que recebeu contribuições de C6 a T1 (SOUZA et al., 2010). A formação de três troncos correspondeu ao encontrado na maioria dos mamíferos domésticos e silvestres (PATERSON, 1887; FIORETTO et al., 2003; SANTANA ET al., 2003; CRUZ \& ADAMI, 2010), com exceção de chinchila (GAMBA et al., 2007) e lobo-marinho (SOUZA et al., 2010) (Tabela 1), que possuem quatro troncos; de porco-espinho com apenas dois troncos (AYDIN, 2003), enquanto em pacas os autores constataram a inexistência de troncos (SCAVONE et al., 2008).

Os nervos peitorais (Nn.P) de tamanduámirim receberam contribuições de todos os troncos do $\mathrm{PB}$, ao contrário do descrito em babuínos, cujos nervos originaram-se dos troncos cranial e médio, com contribuição adicional do NTL (BOOTH et al., 1997) e em chinchilas (GAMBA et al., 2007), que se originaram de apenas três dos quatro troncos presentes. 
Rev. Bras. Saúde Prod. Anim., Salvador, v.13, n.3, p.712-719 jul./set., 2012 http://www.rbspa.ufba.br ISSN 15199940

Tabela 1. Origem dos nervos derivados do plexo braquial a partir dos troncos nervosos de tamanduá-mirim (Tamandua tetradactyla)

\begin{tabular}{lll}
\hline Troncos & Nervos & Território de inervação \\
\hline Cranial & $\begin{array}{l}\text { Supra-escapular (NSP) } \\
\text { Subescapular (NSB) }\end{array}$ & $\begin{array}{l}\text { Músculo supra-espinhoso } \\
\text { Músculo subescapular }\end{array}$ \\
\hline \multirow{2}{*}{ Cranial e Médio } & Axilar (NA) & $\begin{array}{l}\text { Músculos deltoide, infra-espinhoso, } \\
\text { redondo maior e menor } \\
\text { Músculos bíceps braquial, } \\
\text { coracobraquial e braquial }\end{array}$ \\
& Musculocutâneo (NMC) & $\begin{array}{l}\text { Músculo cutâneo do tronco } \\
\text { Múdio e Caudal } \\
\text { Mósculo grande dorsal }\end{array}$ \\
& Torácico Lateral (NTLat.) \\
& Toracodorsal (NTD) & Músculos peitorais \\
\hline \multirow{2}{*}{ Todos } & Radial (NR) & $\begin{array}{l}\text { Músculos: tensor da fáscia do } \\
\text { antebraço, ancôneo, tríceps braquial } \\
\text { Antebraço } \\
\text { Porção medial do tríceps braquial e } \\
\text { antebraço }\end{array}$ \\
\hline
\end{tabular}

O nervo supra-escapular (NSP), no tamanduá-mirim, assemelhou-se em sua origem com o descrito em babuíno (BOOTH et al., 1997), capivara (FIORETTO et al., 2003), porco-espinho (AYDIN, 2004), cateto (MOURA et al., 2007), paca (SCAVONE et al., 2008), lobo-marinho (SOUZA et al., 2010) e macaco-barrigudo (CRUZ \& ADAMI, 2010), e recebeu contribuição essencialmente dos nervos que compuseram o tronco cranial do plexo. Diferiu, na origem e no território de inervação, quando comparado com a chinchila, que recebeu contribuição somente de C6 e supriu os músculos supra-espinhoso, infra-espinhoso e parte do deltoide (GAMBA et al., 2007), enquanto em tamanduá-mirim esse nervo supriu apenas o músculo supra-espinhoso (Tabela 1).

A origem dos nervos subescapulares (NSB) de tamanduá-mirim diferenciou-se daquela da paca, que só recebeu contribuição de C6 (SCAVONE et al., 2008). Porém, em ambas as espécies, o território de inervação foi o músculo subescapular. Na capivara, a origem foi semelhante, com exceção de alguns animais que receberam a contribuição adicional de C7 (FIORETTO et al., 2003).

Em tamanduá-mirim o nervo toracodorsal (NTD) recebeu contribuição dos três troncos do PB, diferentemente do observado em algumas espécies de animais de fazenda (caprinos, ovinos, bovinos, suínos e eqüinos) que apresentaram origem variável, porém com contribuição marcante de C8 em todas as espécies (MAGILTON et al., 1968). Em capivara, houve variação na origem entre os antímeros, isto é, o direito foi mais cranial (FIORETTO et al., 2003), e, em veado, a origem variou com o sexo (MELO et al., 2007). O território de inervação do NTD, tanto em tamanduá-mirim, quanto nos animais já referidos foi o músculo grande dorsal.

A origem dos troncos cranial e médio do nervo axilar (NA) em tamanduá-mirim correspondeu à de babuíno (BOOTH et al., 1997) e à de porco-espinho (AYDIN, 2004). A ramificação para os músculos também se aproximou com o observado em babuíno, de modo a suprir os músculos redondo maior e menor $\mathrm{e}$ 
deltoide, com exceção do músculo infraespinhoso (BOOTH et al., 1997).

$\mathrm{O}$ nervo cutâneo medial do antebraço (NCMA) foi o único que se originou antes da formação dos troncos com contribuição unissegmentar de $\mathrm{T} 1$ e ramificou-se na fáscia do antebraço, diferente do observado em babuíno (BOOTH et al., 1997) que se originou de um tronco comum com os nervos mediano e ulnar, entretanto, apresentou o mesmo território de inervação com o observado em tamanduá-mirim (Tabela 1).

Em paca, o território de inervação do nervo radial (NR) correspondeu ao observado no tamanduá-mirim, porém com origem distinta (SCAVONE et al., 2008). Em chinchila, (GAMBA et al., 2007) e lobo-marinho (SOUZA et al., 2010) a origem desse nervo foi constatada a partir dos troncos médio e caudal e, em porco-espinho (AYDIN, 2004), somente do tronco caudal. Em tamanduá-mirim, a origem assemelhou-se com babuíno (BOOTH et al., 1997) e macaco-barrigudo (CRUZ \& ADAMI, 2010), com contribuição de todos os troncos do plexo braquial, porém com território de inervação distinto.

Para o nervo musculocutâneo (NMC) Melo et al. (2007), chamaram a atenção quanto à variação da origem entre as diversas espécies, sexo e até antímeros. Em chinchila, este nervo originou-se de C6 e C7, supriu os músculos bíceps braquial, braquial e seguiu para a pele do antebraço através do NCMA (GAMBA et al., 2007). Em porco espinho (AYDIN, 2004) e lobo-marinho (SOUZA et al., 2010) o NMC originou-se de um tronco comum com o NM, e em ambas as espécies diferiram do observado em tamanduá-mirim, no qual o NMC apresentou origem a partir dos troncos cranial e médio e supriu, além dos músculos bíceps braquial e braquial, o músculo coracobraquial.
Em chinchila (GAMBA et al., 2007), porco-espinho (AYDIN, 2004) e lobomarinho (SOUZA et al., 2010) o nervo mediano (NM) apresentou origem comum com o nervo ulnar e seguiu para o antebraço sem emitir ramos para o braço, da mesma forma como o observado em tamanduá-mirim.

Em porco-espinho (AYDIN, 2004), babuíno (BOOTH et al., 1997) e lobomarinho (SOUZA et al., 2010) o nervo ulnar (NU) originou-se do tronco caudal e não emitiu ramos para o braço, comportamento distinto do descrito em tamanduá-mirim, cujo nervo originou-se de todos os troncos. O território de inervação em chinchila (GAMBA et al., 2007), paca (SCAVONE et al., 2008), porco-espinho (AYDIN, 2004), capivara (FIORETTO et al., 2003) e lobo-marinho (SOUZA et al., 2010) correspondeu à musculatura do antebraço, conforme o observado em tamanduá-mirim.

$\mathrm{O}$ território de inervação do nervo torácico lateral (NTL) em tamanduámirim foi o músculo cutâneo do tronco, semelhante ao descrito em capivara, porém nessa espécie também emitiu ramo para o músculo peitoral profundo (FIORETTO et al., 2003). Quanto à origem, esse nervo recebeu contribuição mais cranial em veado macho entre C5T1 (MELO et al., 2007) e em babuíno entre C5-C7 (BOOTH et al., 1997), mas em chinchila (GAMBA et al., 2007) e em lobo-marinho (SOUZA et al., 2010) a origem (C8 e T1) assemelhou-se ao descrito em tamanduá-mirim que recebeu contribuição adicional do tronco médio (C7).

O plexo braquial de tamanduá-mirim originou-se dos nervos espinhais C5 a T1 por meio dos troncos: cranial, médio e caudal. Os nervos derivados formaram-se com a participação de um, dois ou três troncos, e a maioria dos nervos apresentou origem e trajeto semelhante 
Rev. Bras. Saúde Prod. Anim., Salvador, v.13, n.3, p.712-719 jul./set., 2012 http://www.rbspa.ufba.br ISSN 15199940

ao plexo braquial de outros mamíferos domésticos e silvestres.

\section{REFERÊNCIAS}

AMORIM JÚNIOR, A.A; AMORIM, M.J.A.A.L.; SILVA, D.R.; PIMENTEL, D.S.; ARAÚJO, F.P.; ALVIM, M.M.S. Origem do plexo braquial no bichopreguiça (Bradypus variegatus Shinz, 1825). International Journal of Morphology, v.21, n.1, p.45, 2003.

AYDIN, A. Brachial plexus of the porcupine (Hystrix cristata). Veterinary Medicine Czech, v.48, n.10, p.301-304, 2003.

AYDIN, A. Nerves originating from brachial plexus of the porcupine (Hystrix cristata). Veterinary Medicine Czech, v.49, n.4, p.123-128, 2004.

BIELIK, P. Constituição do Plexus Brachialis no Bradypus tridactylus. Arquivos de Anatomia e Antropologia, v.18, p.1-4, 1937.

BOOTH, K.K.; AMORIM, M.J.A.A.L.; SILVA, D.R.; PIMENTEL, D. S.; ARAÚJO, F.P.; ALVIM, M.M.S. The brachial plexus in the Chacma baboon (Papio ursinus). Journal of Medical Primatology, v.26, p.196-203, 1997.

CRUZ, G.A.M.; ADAMI, M. Anatomia do plexo braquial de macaco-barrigudo (Lagothrix lagothricha). Pesquisa Veterinária Brasileira, v.30, n.10, p.881886, 2010.

FIORETTO, E.T.; SOUZA CASTRO, M.F.; GUIDI, W.L.; MAINARDI, R.; SOUZA, R.; RIBEIRO, A.A.C.M. Gross anatomic organization of the capybara's (Hydrocharis hydrochaeris) brachial plexus. Anatomy, Histology, Embryology, v.32, n.3, p.169-174, 2003.
GAMBA, C.O.; CASTRO, T.F.; RICKES, E.M.; MARTINEZ, M.A.P. Sistematização dos territórios nervosos do plexo braquial em chinchila (Chinchilla lanigera).

Revista Brasileira de Veterinária de Pesquisa e Ciência Animal, v.44, n.4, p.283-289, 2007.

\section{INTERNATIONAL COMMITTEE ON VETERINARY GROSS ANATOMICAL NOMENCLATURE. Nomina anatomica veterinaria. 5th ed. Hannover, Columbia, Gent, Sapporo: Editorial Committee, 2005. 166p.}

\section{IUCN 2011. IUCN Red List of}

Threatened Species. Version 2011.1. <www.iucnredlist.org>. Acesso em: 15 março 2011.

MAGILTON, J.H.; GETTY, R.; GHOSHAL, N.G. A comparative morphological study of the brachial plexus of domestic animals (Goat, sheep, ox, pig and horse). Iowa State Journal of Science, v.42, p.245-279, 1968.

MEDRI, I.M.; MOURÃO, G.M.; RODRIGUES, F.H.G. Ordem Xenarthra. IN: REIS, N.R.; PERACCHI, A.L.; PEDRO, W.A.; LIMA, I.P. Mamíferos do Brasil. Londrina: Nélio R. dos Reis, 2006.

MELO, S.R. GONÇALVES, A.F.N.; SASAHARA, T.H.C.; FIORETTO, E.T.; GERBASI, S.H.; MACHADO, M.R.; GUIMARÃES, G.C.; RIBEIRO, A.A.C.M. Sex-related Macrostructural Organization of the Deer's Brachial Plexus. Anatomia, Histologia, Embryologia, v.36, n.4, pp.295-299, 2007.

MIRANDA, F.; COSTA, A.M. Xenarthra (tamanduá, tatu, preguiça). In: CUBAS, Z.S.; SILVA, J.C.R; CATÃO-DIAS, J.L. Tratado de animais selvagens: medicina veterinária. São Paulo: Roca, 2006. p.402414. 
MOURA, C.E.B.; ALBUQUERQUE, J.F.G.; MAGALHÃES, M.S.; SILVA, N.B.; OLIVEIRA, M.F.; PAPA, P.C. Análise comparativa da origem do plexo braquial de catetos (Tayassu tajacu).

Pesquisa Veterinária Brasileira, v.27, n.9, p.357-362, 2007.

PATERSON, A.M. The limb plexuses of mammals. Journal of Anatomy and

Physiology, v.21, p.611-634, 1887.

RIBEIRO, A.R.; PRADA, I.L.S.; SILVA, Z.; BARROS, R.A.C.; SILVA, D.C.O.

Origem do plexo braquial do macaco

Cebus apella. Revista Brasileira de

Veterinária de Pesquisa e Ciência

Animal, v.42, n.2, p.143-149, 2005.

SANTANA, J.J.; ALBUQUERQUE, J.F.G.; MOURA, C.E.B.; COSTA, W.B.; OLIVEIRA, M.F.; BARRETO JÚNIOR, R.A.; MIGLINO, M.A. Origem do plexo braquial de mocós (Kerodon rupestris

Wied, 1820). Revista Brasileira de Veterinária de Pesquisa e Ciência Animal, v.40, p.391-396, 2003.
SCAVONE, A.R.F.; MACHADO, M.R.F.; GUIMARÃES G.C.; OLIVEIRA, F.S.; GERBASI, S.H.B. Análise da origem e distribuição dos nervos periféricos do plexo braquial da paca (Agouti paca, Linnaeus, 1766). Ciência Animal Brasileira, v.9, n.4, p.1046-1055, 2008.

SOUZA, D.A.S.; CASTRO, T.F.; FRANCESCHI, R.C.; SILVA FILHO, R.P.; PEREIRA, M.A.M. Formação do plexo braquial e sistematização dos territórios nervosos em membros torácicos de lobos-marinhos Arctocephalus australis. Revista Brasileira de Veterinária de Pesquisa e Ciência Animal, v. 47, n.2, p.168-174, 2010.

Data de recebimento: 17/02/2012

Data de aprovação: 20/06/2012 